\title{
Gradhiva
}

\section{Mario Rispoli, Jean-Charles Depaule, Italien du Caire. Une autobiographie}

Marseille, Éditions Parenthèses-Maison méditerranéenne des sciences de l'homme, coll. « Parcours méditerranéens » 6, 2010

\section{Giordana Charuty}

\section{(2) OpenEdition}

1 Journals

Édition électronique

URL : http://journals.openedition.org/gradhiva/2223

DOI : $10.4000 /$ gradhiva.2223

ISSN : $1760-849 X$

Éditeur

Musée du quai Branly Jacques Chirac

Édition imprimée

Date de publication : 30 novembre 2011

Pagination : 263-265

ISBN : 978-2-35744-046-3

ISSN : 0764-8928

Référence électronique

Giordana Charuty, « Mario Rispoli, Jean-Charles Depaule, Italien du Caire. Une autobiographie », Gradhiva [En ligne], 14 | 2011, mis en ligne le 30 novembre 2011, consulté le 21 septembre 2020. URL : http:// journals.openedition.org/gradhiva/2223; DOI : https://doi.org/10.4000/gradhiva.2223

Ce document a été généré automatiquement le 21 septembre 2020.

(c) musée du quai Branly 


\section{Mario Rispoli, Jean-Charles Depaule, Italien du Caire. Une autobiographie}

Marseille, Éditions Parenthèses-Maison méditerranéenne des sciences de l'homme, coll. « Parcours méditerranéens » 6, 2010

\section{Giordana Charuty}

\section{RÉFÉRENCE}

Mario Rispoli, Jean-Charles Depaule, Italien du Caire. Une autobiographie, Marseille, Éditions Parenthèses-Maison méditerranéenne des sciences de l'homme, coll. «Parcours méditerranéens » 6, 2010, 252 p. 
1

«Une odeur, des sonorités, une qualité de l'air, des luminosités, qu'on m'avait “parlées”, qu'on m'avait montrées, que j'avais rêvées...»(p.11): telle est la matière sensible du pays des origines que retrouve Mario Rispoli à treize ans, en débarquant à Brindisi pour des vacances avec ses copains de l'école Don Bosco du Caire qui, eux, célèbrent l'Année sainte. Dans la capitale égyptienne de l'immédiat après-guerre, l'Italie est présente de bien des façons, plus ou moins antagonistes. Dans l'entre-soi d'une famille napolitaine transplantée depuis un demi-siècle, il y a les récits de la lignée maternelle, installée en Égypte depuis l'époque des grands travaux sur le Nil mais qui se souvient de l'entrée de Garibaldi à Naples, d'avoir crié "Vive la République» sous le règne d'Umberto ${ }^{\text {er }}$ et qui ne supporte pas davantage la confrontation directe avec le régime fasciste. Il y a la mémoire d'un père né à Alexandrie de parents venus de Caserte et de Sicile, puis élevé dans le quartier juif du Caire, et mort trop jeune. Il y a l'école des sœurs franciscaines rue Antikhana, les films projetés dans les jardins de cinémas arabes - le Kursaal, le Nasr - et les amis que retrouve le grand-père au café Stambelos; les chansons patriotiques de la Grande Guerre et le répertoire lyrique que l'on joue et chante à la maison avant de l'écouter à l'opéra; le dialecte napolitain et les accents régionaux du marché du Maarouf; la provola affumicata et le provolone piccante achetés « chezla Triestine » du passage Green pour rehausser la saveur des maccarune $e$ carne etde la pasta e fagioli.

Dans l'Égypte des années 1950, cette communauté est fortement stratifiée. Si, à distance $\mathrm{du}$ fascisme, elle a pu cultiver au sein d'une bourgeoisie d'affaires « une fierté démesurée d'être italiens » (p.99), la lignée maternelle, qui se moque de ceux qui sont « devenus arabes », n'a elle-même aucun lien avec la haute société italienne. Et, pour les fonctionnaires et entrepreneurs qui arrivent alors en Égypte, tous ces Italiens d'" avant " ne sont-ils pas devenus des «bâtards arabisés» (p.54)? Ce n'est qu'en assumant la responsabilité du bureau local de l'ANSA, l'agence de presse italienne, où il a appris le métier de rédacteur, que Mario Rispoli pourra être crédité d'un cosmopolitisme valorisé, cependant qu'il conservera tout au long d'une très active carrière de journaliste ses liens professionnels avec l'Italie, au bureau parisien de l'agence puis au service de presse de la filiale française de Fiat.

3 À son tour, l'univers qu'ordonnent les étages de l'immeuble où il a grandi enfant, rue Malika-Farida, est un vertigineux kaléidoscope de langues, d'histoires, de statuts sociaux, d'identités nationales, d'affiliations religieuses et d'appartenances culturelles. Juifs italiens et arabophones, Grecs orthodoxes, coptes, catholiques de rite byzantin, Maltais, Arméniens, Palestiniens, Yougoslaves composent les " gens du pays » face aux quelques Européens occidentaux, Britanniques, Français, Italiens. Chaque famille, en outre, dispose de plusieurs langues, parlées et écrites, pour différencier toutes les 
nuances du dedans et du dehors. À l'image des conteurs de Nagib Mahfûz, dépositaires de la mémoire d'un quartier, le narrateur se remémore, avec une remarquable minutie, une succession de microcosmes qu'adolescent il pouvait, somme toute aisément, franchir d'un étage à l'autre, d'un balcon à l'autre, d'une rue à l'autre : pour passer de la très européenne rue Malika-Farida à la vie indigène des ruelles adjacentes, quelques heures d'école buissonnière suffisaient. Et l'on devine tout ce que la belle évocation des univers sonores de chacun de ces mondes sociaux doit à l'écoute sensible d'un interlocuteur pour qui l'anthropologie de la vie urbaine est un art éclairé de la rencontre.

4 Bien loin, en effet, de reconduire une vision enchantée du cosmopolitisme du Caire, ce portrait du khawâga Mario - terme tour à tour prestigieux et dépréciatif désignant habituellement l'étranger non musulman, issu de minorités locales (p. 244, 245) entend répondre à de très précises questions sociologiques qui sont traitées, chapitre après chapitre, selon une composition thématique où les expériences d'une vie rencontrent celles qui font la grande Histoire. À la formation au sein de l'univers familial, au répertoire des "figures héroïques de tout acabit» des idéaux de l'adolescence et de la jeunesse (p. 247), succède le double apprentissage du métier de journaliste de la presse écrite et radiophonique et d'une éducation sentimentale ouvrant sur le choix, ô combien incertain au sein d'une pareille mosaïque culturelle, d'une compagne de vie socialement convenable. Mais, après les événements de 1956, l'apprenti rédacteur d'agence qui se découvre " en Égypte » prendra conscience d'avoir vécu, non tant dans une société multiethnique et pluriconfessionnelle, que «dans le refus du pays et de ses habitants ». L'injonction de ne pas faire « comme les Arabes » et l'ignorance des réalités égyptiennes reviennent, en effet, comme un leitmotiv dans l'évocation de cette adolescence à l'européenne pourtant confrontée à de multiples autres. Et, en entrant à sa suite dans ces communautés de métier qui assurent le travail d'information politique, le lecteur pénètre dans un tout autre univers cosmopolite: l'extravagante diversité linguistique de Radio Le Caire, rue Chérifeyn, d'où l'Égypte diffuse des émissions en plus d'une soixantaine de langues, du grec au swahili en passant par l'hébreu, outre l'arabe, l'anglais, le français, l'italien, l'allemand, etc. Là se croisent, dans " un luxueux désespoir », des exilés d'Afrique, des universitaires russes, des réfugiés politiques de tous les mouvements de libération nationale: «une génération sans suite, à la charnière entre le monde colonial et aristocratique et le Tiers Monde militant, qui perdait les guerres, gagnait les combats politiques et créait le bloc des non-alignés » (p. 145).

5 La trame thématique, de fait, n'efface pas toute construction linéaire. Elle donne, au contraire, à chaque période de la vie plus de résonance et plus de densité. Ainsi, les deux chapitres consacrés aux guerres peuvent-ils mettre en perspective des vécus à première vue incommensurables : par exemple, l'exil intérieur du jeune Italien passant brusquement, en 1956, du statut d'étranger privilégié à celui d'étranger toléré, alors que le patriotisme égyptien devient une composante centrale du nationalisme arabe et, en revanche, la joie intérieure pour la victoire israélienne qui, au lendemain de la guerre des Six Jours en juin 1967, plonge dans le désespoir Nasser et son peuple. Ou encore, l'improbable opération Badr qui, en octobre 1973, déclenche la guerre du Kippour, conduisant à l'arrivée au Caire, parmi les journalistes venus du monde entier, des meilleurs confrères italiens. Et ce seront en fait les anciennes amitiés adolescentes 
avec les jeunes scouts des Éclaireurs de France qui, au dire même du narrateur, vont favoriser les sympathies pro-israéliennes du rédacteur de l'ANSA.

Parmi toutes les questions que pose cette riche trajectoire, il importe de comprendre l'indéfectible attachement à la langue et à la culture françaises produit par la fréquentation du lycée de la Mission laïque française, Bab El-Louk, et cette expérience de scoutisme chez les Éclaireurs de France, qui pouvaient faire contrepoids à une culture catholique napolitaine transplantée au cœur de la capitale égyptienne. Une culture incorporée dont des pans entiers peuvent resurgir, des dizaines d'années plus tard, comme dans cette longue remémoration de toutes les expériences de la mort qui font sens dans une vie. Elle compose un dernier chapitre - Carica di lacrime e di lutti («Chargée de larmes et de deuils») - où s'impose la langue italienne, celle du grand poète Salvatore Quasimodo, pour restituer l'étonnante cohabitation d'anciennes et de plus modernes relations aux morts, familiaux et étrangers.

Dans une belle postface, Jean-Charles Depaule revient, avec la pudeur de l'amitié et la vigilance du chercheur, sur le «compromis d'écriture» que fut cette autobiographie dialogique, une composition à plusieurs mains du portrait en miroir d'un homme et d'un monde, ou plutôt de plusieurs mondes. Un récent numéro de L'Homme proposaitdedistinguer trois joueurs dans tout récit de vie : celui qui a traversé une série d'événements, celui qui fait retour, en position de décalage, sur ce vécu et celui qui devient le narrateur de cette double matière biographique ${ }^{1}$. Dans l'expérimentation qui nous est proposée ici, comment cette partie à trois s'est-elle distribuée ? Comment le «biographant » et le « biographe » sont-ils passés des transcriptions d'entretiens et des archives personnelles du «biographié » aux chapitres que nous lisons? Jean-Charles Depaule précise la manière dont les transcriptions des entretiens enregistrés ont relancé, pour l'un et l'autre, le travail d'enquête dans la mémoire, les "papiers » personnels, les instruments de vérification; comment elles ont nourri toutes sortes d'échanges de textes; comment elles sont, finalement, parvenues au portrait très écrit que nous lisons, après le passage de chaque chapitre par l'épreuve d'un retour à l'oralité à travers une lecture à haute voix qui équivaut, peut-on penser, à cette voix intérieure de la remémoration qui fut l'objet de l'enquête (p. 236, 237). Ce que, dans un article précédent, l'anthropologue a résumé par une formule lapidaire: «Dans un mouvement fait de lectures à haute voix, de questions et de réponses, de suggestions, d'objections, d'ajouts et de retranchements, s'est écrit l'autoportrait d'un Italien du Caire $^{2}$.»

8 De fait, la précision des données sociologiques et historiques ne compose pas un contexte extérieur à la narration biographique; pour motiver l'aléatoire d'une trajectoire singulière, l'appréhension sensible des choses et des gens n'est jamais dissociée du travail interprétatif et de l'imaginaire qui leur donnent sens. Plus que jamais se trouve affirmé ici le souci, maintes fois déclaré par le chercheur, de restituer des identités "circonstancielles ", «contextuelles». Aussi est-ce la métaphore du cadrage photographique et de ses variations qui, finalement, impose sa pertinence pour rendre compte du minutieux travail de composition qui fait la belle réussite de ce livre. 


\section{NOTES}

1. Daniel Fabre, Jean Jamin et Marcello Massenzio, «Jeu et enjeu ethnographiques de la biographie ", L'Homme 195-196, 2010, p. 14, 15.

2. Jean-Charles Depaule, «Personnes de rencontre », Cahiers internationaux de sociologie 124, 2008 p. 188.

\section{AUTEURS}

\section{GIORDANA CHARUTY}

giordana.charuty@laposte.net 